\title{
Attosecond Circular Dichroism Spectroscopy of Polyatomic Molecules
}

\author{
Olga Smirnova, ${ }^{1}$ Serguei Patchkovskii, ${ }^{1}$ Yann Mairesse, ${ }^{2}$ Nirit Dudovich, ${ }^{3}$ David Villeneuve, ${ }^{1}$ \\ Paul Corkum, ${ }^{1}$ and Misha Yu. Ivanov ${ }^{1}$ \\ ${ }^{1}$ National Research Council, 100 Sussex Drive, Ottawa, Ontario K1A OR6, Canada \\ ${ }^{2}$ CELIA, Université Bordeaux I, UMR 5107 (CNRS, Bordeaux 1, CEA), 351 Cours de la Libération, 33405 Talence Cedex, France \\ ${ }^{3}$ Department of Physics of Complex Systems, Weizmann Institute of Science, Rehovot 76100, Israel
}

(Received 24 May 2008; published 9 February 2009)

\begin{abstract}
We describe the roles of multiple electronic continua in high-harmonic generation from aligned molecules. First, we show how the circularity of emitted harmonics tracks the interplay of different electronic continua participating in the nonlinear response. Second, we show that the interplay of different continua can lead to large variations of harmonic phases. Finally, we show how multiple electronic continua allow one to shape the polarization of high harmonics and attosecond pulses.
\end{abstract}

PACS numbers: $42.50 . \mathrm{Hz}, 33.80 . \mathrm{Wz}$

High-harmonic generation (HHG) is a new type of nonlinear optical spectroscopy emerging at the boundaries of nonlinear optics, attosecond, and strong-field physics [1,2]. HHG spectroscopy observes harmonics of the incident laser radiation, emitted when an electron liberated from a molecule by an intense laser field recombines with the parent ion. It promises to yield structural $[1,2]$ information about the molecule, encoded in the so-called "structural" minima in the harmonic spectra [2]. These minima occur due to the destructive interference in the recombination to multiple "atomic" centers of the same molecular orbital. The major interest in the analysis of such minima in HHG spectra (e.g., [2-9]) underscores their critical importance for HHG imaging. The interplay of experimental parameters-laser intensity, molecular alignment, saturation of ionization-can affect the positions of these minima $[3,4,8]$, making quantitative analysis especially important. HHG spectroscopy can also be used to monitor nuclear dynamics triggered by ionization [10-12], opening the route to combining attosecond temporal and sub- $\AA$ spatial information.

Not only nuclear, but also much faster-electronicdynamics can be excited by strong-field ionization, which can leave the molecular ion in various electronic states $[13,14]$. Different ionization channels give rise to different HHG channels. Destructive interference between them also leads to minima in the harmonic spectra. These "dynamical" minima are not related to the structure of molecular orbitals, but reflect electronic dynamics in the ion between ionization and recombination $[13,14]$ —ionization into a number of ionic states launches an electron-hole wave packet. Thus, disentangling structural and dynamical information in the harmonic spectra is the key problem in attosecond dynamical imaging.

We use the example of a $\mathrm{CO}_{2}$ molecule to show how the interplay of different HHG channels is encoded in the polarization of emitted harmonics. We have found that, for linearly polarized incident laser radiation, dynamical minima in the harmonic spectra lead to circular dichroism in high-harmonic emission. As opposed to structural minima, the position of the dynamical minima can be controlled by changing the peak laser intensity. This property opens a route to controlling the ellipticity and the sense of rotation of attosecond pulses produced via HHG, solving a difficult problem of controlling light polarization in the XUV range. We also show that the dynamical minima can lead to large phase variations in the harmonic phases, which can be similar to those found for the structural minima. Sensitivity to laser intensity distinguishes the two.

Circular dichroism means that a system responds differently to left and right circularly polarized light, revealing the lack of left-right symmetry. Linearly polarized light is a sum of left and right circular light. Thus, circular dichroism can be detected by sending a linearly polarized laser pulse and measuring the polarization of emitted or scattered light. The corresponding parameter is the circularity $m_{C}$ : $m_{C}=\left[\left|E_{R}\right|^{2}-\left|E_{L}\right|^{2}\right] /\left[\left|E_{R}\right|^{2}+\left|E_{L}\right|^{2}\right]$, where $\left|E_{R}\right|$ and $\left|E_{L}\right|$ are the amplitudes of right and left polarized fields. It can be also expressed as $m_{C}=2 \operatorname{Im}\left(E_{x}^{*} E_{z}\right) /\left(\left|E_{x}\right|^{2}+\right.$ $\left|E_{z}\right|^{2}$ ), where the $x-z$ plane is the plane of polarization of the emitted light.

We define the $x-z$ plane by the main axis of the aligned molecular ensemble and the laser polarization; the $z$ axis is chosen parallel to the laser polarization. Left-right symmetry is absent when the ensemble is aligned at an angle $\Theta \neq 0^{\circ}, 90^{\circ}$ relative to the laser polarization. Circular dichroism in the high-harmonic emission can originate already from the anisotropy of the scattering potential, which affects the motion of the returning electron. Nevertheless, high harmonics are typically linearly polarized: the electron moves predominantly along the strong laser field and the effect of the anisotropy is small. To achieve high circularity, two conditions have to be met. First, the typically dominant parallel component of the harmonic emission has to be suppressed, making it comparable to the emission perpendicular to the laser field. Second, the relative phase between the two components has to approach $\pi / 2$. These 
conditions are naturally met in the vicinity of both structural and dynamical minima.

Indeed, due to the anisotropy of the ionic potential, the destructive interference-whether structural or dynamical-cannot occur simultaneously in parallel $(z)$ and perpendicular $(x)$ components of the harmonic light. Since the parallel component generally dominates, destructive interference in the parallel light makes the $z$ and $x$ components comparable, meeting the first condition for high circularity. Second, both for structural and, as we show below, for dynamical minima, the phase of the parallel component changes by $\sim \pi$ across the minimum. Therefore, the relative phase between the two components inevitably passes through $\pi / 2$, meeting the second condition for high circularity.

To describe the dynamics of polyatomic molecules in strong infrared laser fields, we combine the methods of quantum chemistry and strong-field physics. Harmonic generation results from laser-induced polarization $\mathbf{D}(t)$. Generalizing [15], we write $\mathbf{D}(t)$ (in atomic units) as:

$$
\begin{aligned}
\mathbf{D}(t, \Theta) \propto & \sum_{j, t_{b}} a_{\text {ion, }, j}\left[t_{b}(t), \Theta\right] a_{\text {prop }, j}\left[t, t_{b}(t), \Theta\right] \\
& \times\left\langle\Psi_{N T}^{(N)}(t, \Theta)|\hat{\mathbf{d}}| \hat{A} \Psi_{j}^{(N-1)}(t, \Theta) \Phi_{C, j}(t, \mathbf{k}(t), \Theta)\right\rangle .
\end{aligned}
$$

The $N$-electron wave function $\Psi_{N T}^{(N)}$ describes the neutral molecule, including depletion by ionization. $\Psi_{j}^{(N-1)}$ describes dynamics of the ion from ionization at $t_{b}=t_{b}(t)$ until recombination at $t$, including laser-induced transitions between the ionic states; $j$ labels the initial ionic state. The relationship between $t_{b}(t)$ and $t$ includes the effect of the ionic potential, see [16]. The amplitude of each HHG channel $j$ includes ion-state-specific subcycle ionization amplitude $a_{\text {ion }, j}\left(t_{b}\right)$ and the propagation amplitude $a_{\mathrm{prop}, j}$ between $t_{b}$ and $t$. Continuum electron is described by the scattering state $\Phi_{C, j}$, correlated to the state of the ion and characterized by the (asymptotic) kinetic momentum $\mathbf{k}(t)$ acquired from the laser field. The operator $\hat{A}$ antisymmetrizes the electrons. Angle $\Theta$ characterizes molecular alignment. The harmonic field is calculated by the Fourier transform of Eq. (1) and averaged over the molecular alignment distribution.

Quantum chemistry methods are used to describe the laser-induced dynamics of bound electrons, both in the ion and in the neutral. First, we calculate the multielectron wave functions of the neutral and the ion. The calculations were performed using the GAMESS quantum chemistry code [17], complete active space self-consistent field (CASSCF) method and correlation-consistent valence triple-zeta basis set expansion [18]. In the neutral $\mathrm{CO}_{2}$, the influence of the IR laser field is included quasistatically. In the ion, excited electronic states $\tilde{A}^{2} \Pi_{u}(j=\tilde{A}$, channel $A)$ and $\tilde{B}^{2} \Sigma_{u}^{+}(j=\tilde{B}$, channel $B)$ are close to the ground state $\tilde{X}^{2} \Pi_{g}(j=\tilde{X}$, channel $X)$. To include the multielectron dynamics in the ion, we calculate the transition dipole moments between these field-free states and their quasistatic Stark shifts due to other states. Then, we solve the time-dependent Schrödinger equation in the restricted basis of the states $\tilde{X}, \tilde{A}, \tilde{B}$. For the ionization channel $j$, the initial condition is set by populating the quasistatic state correlated to the field-free state $j$ at $t_{b}(t)$.

Strong-field methods are used to model the continuum dynamics. To find the ionization amplitude, we use the approach of [19]. Ionization, which leaves the ion in an electronic state $j$, is described using the Dyson orbital $\left|\psi_{D, j}\right\rangle=\sqrt{N}\left\langle\psi_{j}^{(N-1)} \mid \psi_{N T}^{(N)}\right\rangle$. To take into account multicenter geometry of the orbital, we write it as linear superposition of spherical atomic orbitals. This representation shows that $a_{\text {ion, },}\left[t_{b}, \theta\right]=a_{\text {ion }, j}^{(0)}\left[t_{b}\right] R_{j}(\Theta)$. Here $a_{\text {ion }}^{(0)}\left[t_{b}\right]$ is the "atomic" ionization amplitude from the single atomic orbital. The term $R(\Theta)$, determined by the interference of the atomic orbitals, is calculated as described in [20]. For $a_{\text {ion }}^{(0)}\left[t_{b}\right]$ we use the subcycle formula [21] with the Coulomb corrections. Expression for the propagation amplitude is standard; see [15].

The scattering state $\Phi_{C, j}$ with asymptotic momentum $k(t)$ is found by substituting the ansatz $\Psi_{C, j}^{(N)}(t, \theta)=$ $\hat{A} \Psi_{j}^{(N-1)}(t, \theta) \Phi_{C, j}(t, \theta) \quad$ into the time-dependent $N$-electron Schrödinger equation. After some algebra this
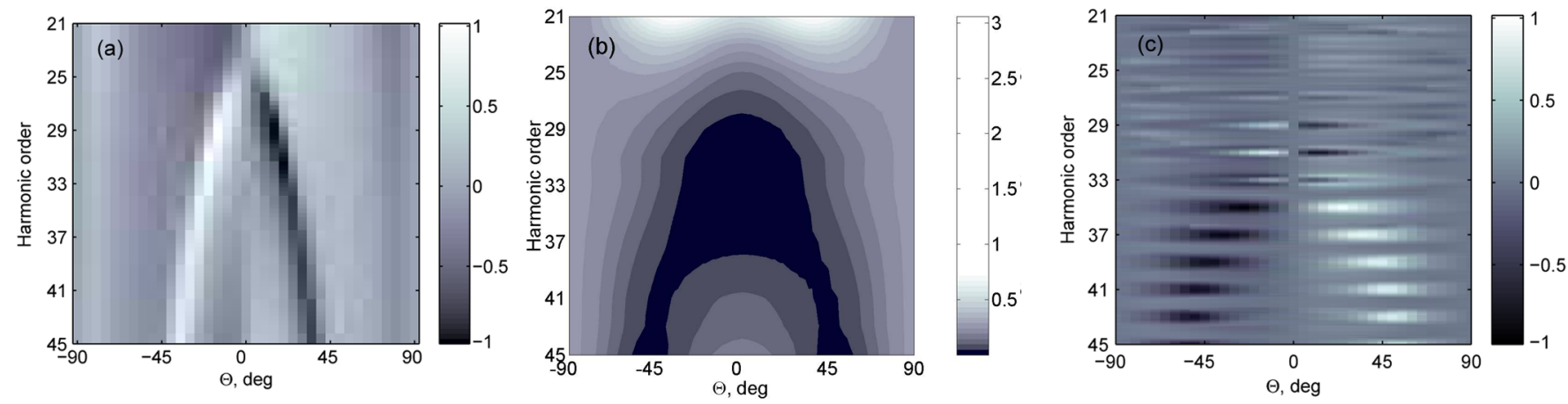

FIG. 1 (color online). (a) Circularity of the recombination dipole for the channel $X$ (no angular averaging); (b) HHG spectrum for different alignment angles, averaged over the alignment distribution; dark color marks amplitude minimum. The laser parameters are $I=2 \times 10^{14} \mathrm{~W} / \mathrm{cm}^{2}, \lambda=800 \mathrm{~nm}$, pulse duration $40 \mathrm{fsec}$. (c) Circularity of the harmonic emission, same laser parameters. 
substitution yields effective one-electron equation for $\Phi_{C, j}$ [22]. The interaction potential includes the laser field, $V_{L}(t)$, and the ionic core, $U_{\text {ion }, j}(t)$. The core potential includes the Coulomb potential of the nuclei and the Hartree potential of the core electrons in the ionic state corresponding to the channel $j$. The nonlocal exchange contributions and the electron-induced core polarization have been omitted. The equation is solved using the approach of [16], which includes the acceleration of the laserdriven electron by the ionic core and removes the ambiguities [5-9] in relating the energy $\Omega$ of the emitted photon to the asymptotic momentum $k$ of the incoming electron, $\Omega=I_{p}+k^{2} / 2$. For an alternative approach to using scattering states in HHG; see [23].

Armed with this approach, we simulate high-harmonic generation in a $\mathrm{CO}_{2}$ molecule. The laser pulse is a $40 \mathrm{fsec}$ Gaussian, the wavelength is $\lambda=800 \mathrm{~nm}$. To reflect typical experimental situation, we suppress the so-called "long" trajectories. The results are averaged over typical experimental alignment distributions (e.g., [6,8,24], characteristic $\Theta \sim 30^{\circ}$ for aligned ensemble; the distribution was modeled as $\left.\cos ^{4} \Theta\right)$.

Figure 1(a) shows $m_{C}$ of the recombination dipole for the single HHG channel $X$ (ion in the ground state $\tilde{X}^{2} \Pi_{g}$ ), without averaging over the alignment distribution. As expected, high $m_{C}$ marks the structural minimum in the recombination dipole. Figure 1(b) shows the full HHG spectrum for $I=2 \times 10^{14} \mathrm{~W} / \mathrm{cm}^{2}$ versus the harmonic number and the alignment angles, after angular averaging. The signal is normalized to $\Theta=90^{\circ}$. Deep minimum occurs at $\mathrm{H} 33$ for $\Theta=0^{\circ}$, consistent with experiment [6]. It shifts to higher harmonic numbers with increasing $\Theta$ (the dark "trenches"). Figure 1(c) shows the map of $m_{C}(\Theta)$ for the same intensity. As expected, the maximal $m_{C}$ indicates the positions of the minima.

The amplitude minimum in Fig. 1(b) has dynamical and not structural origin. Indeed, for parallel alignment of the molecular ensemble, the signal is dominated by molecules aligned at $\sim 35^{\circ}$ thanks, in part, to the dominance of ionization at $45^{\circ}-50^{\circ}$ [24]. For these angles, the structural minimum in the $X$ channel [Fig. 1(a)] appears at H37-39. Thus, angular averaging moves the structural minimum for the $X$ channel to H37-39 for molecular ensemble aligned parallel to the polarization of the probe. The dynamical origin of the minimum in Fig. 1(b) is proved by looking at the harmonic phases for parallel emission, see, e.g., H35 in Fig. 2(a). For $\Theta=0^{\circ}-40^{\circ}$ the phases of the main contributing channels $X$ and $B$ are (i) flat, indicating the absence of structural minima [25], (ii) nearly $\pi$ out of phase, proving that the channels interfere destructively. The total harmonic signal experiences large phase variation $\approx 0.8 \pi$ as $\Theta$ increases [Fig. 2(a)], resembling similar variation for the structural minima [2,9]. Here, the phase variation is due to the switching between the channels; see Fig. 2(b): The amplitude (i.e. the square root of the harmonic intensity) of the channel $B$ decreases with $\Theta$, while the amplitude of the channel $X$ increases. The same channel interplay leads to the $\sim \pi$ phase-variation vs the harmonic number near the dynamical minimum (note the sign change of $m_{C}$ vs the harmonic number in Fig. 1(c). The degree of circular polarization $m_{C}(\Theta)$ is shown in Fig. 2(c) for H35. As expected, $m_{C}$ maximizes at the position of the dynamical minimum $\left(\Theta=15^{\circ}-20^{\circ}\right)$, where the dominant channel switches between $X$ and $B$.

This interplay of different channels is not specific for $\mathrm{CO}_{2}$. It will occur in all molecules where (i) electronic states of the ion are close to the ground state, (ii) the Dyson orbital for the ground state of the ion has nodal planes (e.g., $\Pi$-type HOMO vs deeper $\Sigma$-type orbitals). Ionization along the nodal plane leads to the destructive interference of ionization currents from the opposite sides of the nodal plane $[3,19]$. The same interference also suppresses recombination to HOMO, enhancing the relative contribution of channels with higher $I_{p}$.

Observation of a structural minimum can be difficult. Once the structural minimum occurs in one channel, it is 'filled' by the contribution of other channels and becomes less visible in total spectrum. Such interplay of channels is not necessarily accompanied by the $\sim \pi$ relative phase between the channels. Thus, the phase between the $z$ and
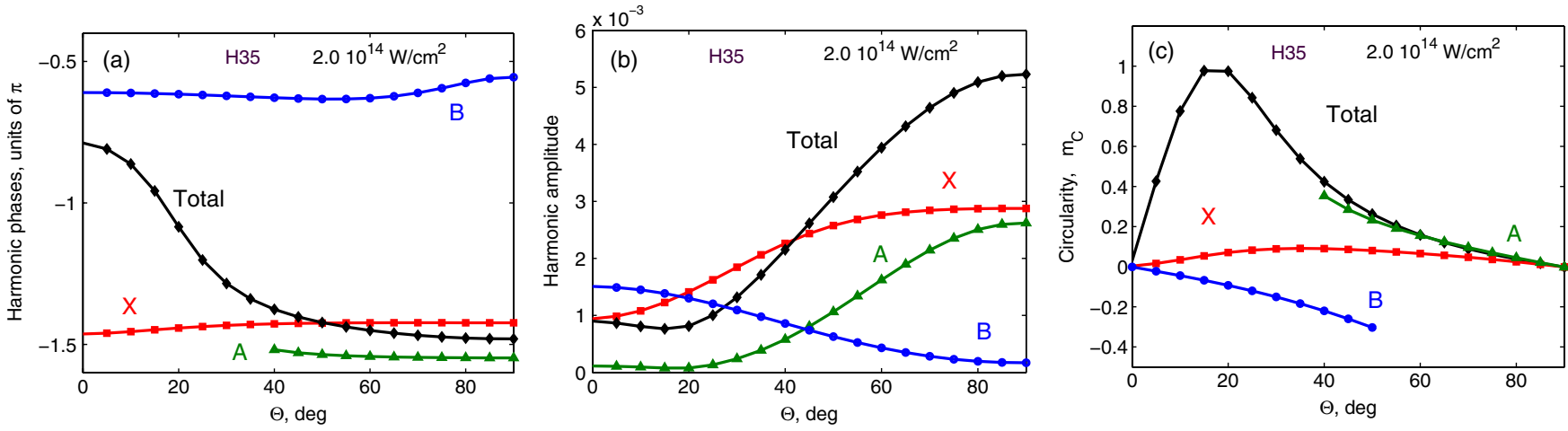

FIG. 2 (color online). Panels (a),(b), and (c) show phases, amplitudes (square root of intensity) and circularity $m_{C}$ of the channels $X$ (red squares), $A$ (green triangles), $B$ (blue circles) and the total signal (black diamonds) for the harmonic H35. Phases and circularity are only shown where channel amplitudes are significant. Laser parameters are the same as in Fig. 1. 

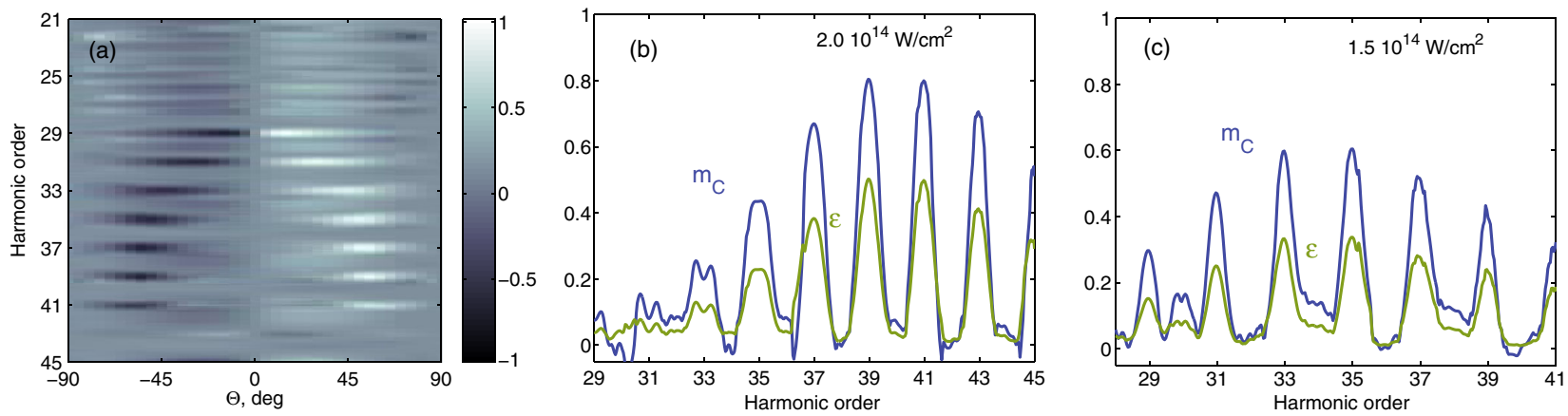

FIG. 3 (color online). Intensity dependence of harmonic polarization. (a) Circularity $m_{C}$ for $I=1.5 \times 10^{14} \mathrm{~W} / \mathrm{cm}^{2}$, compare with Fig. 1(c). Panels (b) and (c) show $m_{C}$ (blue) and $\epsilon$ (green) for $I=2 \times 10^{14} \mathrm{~W} / \mathrm{cm}^{2}$ (b) and $I=1.5 \times 10^{14} \mathrm{~W} / \mathrm{cm}^{2}(\mathrm{c})$, for $\Theta=45^{\circ}$.

$x$ components does not necessarily pass $\pi / 2$, and the harmonics do not become circular.

The key feature that distinguishes a dynamical from a structural minimum and identifies its origin is the intensity dependence [14]. While the dynamical minimum shifts approximately linearly with the laser intensity [14], the position of the structural minimum is fixed by the energy (de Broglie wavelength) of the returning electron and only weakly depends on intensity. The same applies to high $m_{C}$. This intensity dependence is shown in Fig. 3(a) vs Fig. 1(c) and in Fig. 3(b) vs Fig. 3(c), further illustrating the dynamical origin of high $m_{C}$. As we reduce the laser intensity, high values of $m_{C}$ shift to lower harmonic numbers.

If several harmonics have large $m_{C}$, one can synthesize an attosecond pulse or pulse train with controlled $m_{C}$. In $\mathrm{CO}_{2}, m_{C}$ is only significant near the harmonic cutoff. Figures 3(b) and 3(c) show the circularity $m_{C}$ of the cutoff harmonics, for two intensities and $\Theta=45^{\circ}$. Selecting harmonics $\mathrm{H} 37-\mathrm{H} 43$ for $I=2 \times 10^{14} \mathrm{~W} / \mathrm{cm}^{2}$ one can make $\sim 150$ asec pulse with $m_{C} \sim 0.7$.

Current experiments measure the ellipticity of harmonics, which can be expressed as $\epsilon=\left[\left|E_{R}\right|-\left|E_{L}\right|\right] /\left[\left|E_{R}\right|+\right.$ $\left.\left|E_{L}\right|\right]$. While $m_{C}$ counts the ratio of left and right photons, $\epsilon$ compares left and right fields, $\epsilon \leq m_{C}$ [Figs. 3(b) and 3(c)]. Polarization measurements in aligned $\mathrm{CO}_{2}$, performed for harmonics lower than $\mathrm{H} 29$, have not revealed any significant ellipticity [26]. However, the contrast of the polarizers used in these experiments limited the minimum ellipticity that could be measured to a few $10 \%$. We predict $\epsilon<10 \%$ below $\mathrm{H} 29$ for above intensities.

Relative phase between HHG channels is dominated by the attosecond dynamics in the ion between ionization and recombination. The polarization of high harmonics characterizes this dynamics. Strong circular dichroism of HHG in $\mathrm{N}_{2}$ [27] is likely indicating such dynamics. In general, polarization measurements have the advantage of reduced background. Whenever strong channel interference occurs, the polarization measurements will enhance the contrast of the detection. If the amplitude minimum is not accompanied by large ellipticity, it is neither structural nor dynami- cal. It simply reflects switching from one dominant HHG channel to another without destructive interference between them.

We acknowledge stimulating discussions with A. Baltuska, A. Stolow, and the NSERC SRO support.

[1] P. B. Corkum and F. Krausz, Nature Phys. 3, 381 (2007).

[2] M. Lein, J. Phys. B 40, R135 (2007).

[3] Anh-Thu Le, X.-M. Tong, and C. D. Lin, Phys. Rev. A 73, 041402 (2006).

[4] Peng Liu et al., Phys. Rev. A 78, 015802 (2008).

[5] J. Itatani et al., Nature (London) 432, 867 (2004).

[6] C. Vozzi et al., Phys. Rev. Lett., 95, 153902 (2005).

[7] T. Kanai, S. Minemoto, and H. Sakai, Nature (London) 435, 470 (2005).

[8] X. Zhou et al., Phys. Rev. Lett. 100, 073902 (2008).

[9] W. Boutu et al., Nature Phys. 4, 545 (2008)

[10] M. Lein, Phys. Rev. Lett. 94, 053004 (2005).

[11] S. Baker et al., Science 312, 424 (2006).

[12] S. Baker et al., Phys. Rev. Lett., 101, 053901 (2008).

[13] Y. Mairesse et al., Nature Phys. (to be published).

[14] O. Smirnova et al., Nature (London) (to be published).

[15] M. Ivanov, T. Brabec, and N. H. Burnett, Phys. Rev. A 54, 742 (1996).

[16] O. Smirnova, M. Spanner, and M. Yu. Ivanov, Phys. Rev. A, 77, 033407 (2008).

[17] M. W. Schmidt et al., J. Comput. Chem. 14, 1347 (1993).

[18] T. H. Dunning, Jr., J. Chem. Phys. 90, 1007 (1989).

[19] J. Muth-Böhm, A. Becker, and F.H. M. Faisal, Phys. Rev. Lett. 85, 2280 (2000).

[20] Y. Mairesse et al., New J. Phys. 10, 025015 (2008).

[21] G. Yudin and M. Ivanov, Phys. Rev. A 64, 013409 (2001).

[22] S. Tonzani and C. Greene, J. Chem. Phys. 122, 014111 (2005).

[23] Z. Walters, S. Tonzani, and C. Greene, J. Phys. B 40, F277 (2007).

[24] D. Pavicic, Phys. Rev. Lett. 98, 243001 (2007).

[25] Angular averaging moves the phase jump in the $X$ channel beyond H37-39.

[26] J. Levesque et al., Phys. Rev. Lett. 99, 243001 (2007).

[27] X. Zhou et al., Phys. Rev. Lett. (to be published). 